\title{
On the Dynamics of Two-Dimensional Capillary-Gravity Solitary Waves with a Linear Shear Current
}

\author{
Dali Guo, ${ }^{1}$ Bo Tao, ${ }^{1}$ and Xiaohui Zeng ${ }^{2}$ \\ ${ }^{1}$ School of Science, Southwest Petroleum University, Chengdu, Sichuan 610500, China \\ ${ }^{2}$ State Key Laboratory of Oil and Gas Reservoir Geology and Exploitation, Southwest Petroleum University, Chengdu 610500, China \\ Correspondence should be addressed to Bo Tao; taoboo68@gmail.com
}

Received 20 January 2014; Revised 25 March 2014; Accepted 27 March 2014; Published 14 April 2014

Academic Editor: Ricardo Weder

Copyright (c) 2014 Dali Guo et al. This is an open access article distributed under the Creative Commons Attribution License, which permits unrestricted use, distribution, and reproduction in any medium, provided the original work is properly cited.

\begin{abstract}
The numerical study of the dynamics of two-dimensional capillary-gravity solitary waves on a linear shear current is presented in this paper. The numerical method is based on the time-dependent conformal mapping. The stability of different kinds of solitary waves is considered. Both depression wave and large amplitude elevation wave are found to be stable, while small amplitude elevation wave is unstable to the small perturbation, and it finally evolves to be a depression wave with tails, which is similar to the irrotational capillary-gravity waves.
\end{abstract}

\section{Introduction}

A capillary-gravity wave on a fluid surface is influenced by both the effects of surface tension and gravity. The wavelength of capillary-gravity waves in water is typically less than a few centimeters; therefore, they are often referred to as ripples. On the open ocean, much larger surface water waves may result from coalescence of smaller wind-caused capillarygravity waves. In the past several decades, the irrotational capillary-gravity waves have been studied by many scientists. In the beginning stage of the wind-driven wave, shear layer is often generated by the wind where the capillary-gravity waves often run on. So it is more realistic to consider the whole system in the presence of vorticity, and the simplest way is to incorporate a linear background shear in the potential flow.

Steady gravity waves with constant vorticity have been considered numerically by applied mathematicians in 1980s and 1990s. Many new profiles due to the shear effect have been found (see [1-4] and references therein). In the recent ten years, pure mathematicians revisit this problem for both steady and unsteady cases. New Hamiltonian formulation for the modified potential system and model equations under different assumptions have been derived. For the steady problem, Constantin et al. considered quite general distribution of the vorticity in [5-8], while for the unsteady flow, most of the works focus on the constant vorticity, and the read is referred to the references [9-11].

In the numerical aspects of two-dimensional surface water waves, the boundary integral method is proven to be very accurate and efficient for finding permanent wave profiles. For the unsteady computation, a new method based on time-dependent conformal mapping was derived by Dyachenko et al. [12] and widely used by other scientists later on. Choi (2009) generalized this method to incorporate the background linear shear for gravity waves in [13]. Most recently, the dynamics of capillary-gravity solitary waves without background shear are considered by Milewski et al. [14] which is based on the same technique. A survey of the numerical methods for time-dependent irrotational water wave is provided by Dias and Bridges (2006).

In [15], the authors studied the steady capillary-gravity solitary wave propagating at the surface of a fluid of finite depth with constant vorticity, using the asymptotic analysis for small amplitude waves and direct numerical computation for full potential equations. However, the dynamics of these waves have not been studied yet, and in this paper, we try to bridge the gap. The rest of the paper is organized as follows. In Section 2, the governing equation is transferred to be a potential equation under a simple variable substitution. In Section 3, we derive the numerical method for computing 
the full potential surface water waves in the presence of constant vorticity. In Section 4, the main numerical results are presented, including the typical profiles of solitary waves, bifurcation diagrams, and stability analysis.

\section{Mathematical Formulation}

We consider 2D surface water waves and choose Cartesian coordinates so that the $y$-axis points upwards and $x$-axis is the direction of wave propagation. The governing equation of motion is incompressible Euler system as follows:

$$
\begin{gathered}
u_{x}+u_{y}=0 \quad \text { conservation of mass, } \\
u_{t}+u u_{x}+v u_{y}=-P_{x} \quad v_{t}+u v_{x}+v v_{y}=-P_{y}-g
\end{gathered}
$$

conservation of momentum,

where $(u, v)$ is the velocity field according to $x$ and $y$ direction; respectively, $P(t, x, y)$ denotes the pressure and $g$ is the gravitational acceleration. The free boundary conditions for capillary-gravity waves are

$$
\begin{aligned}
& v=\zeta_{t}+u \zeta_{x} \quad \text { kinematic boundary condition, } \\
& P=P_{0}-\frac{\sigma}{\rho} \frac{\zeta_{x x}}{\left(1+\zeta_{x}^{2}\right)^{3 / 2}} \quad \text { dynamic boundary condition, }
\end{aligned}
$$

where the free surface displacement $y=\zeta(t, x)$ is supposed to be a graph of function, constant $\rho$ is the fluid density, $\sigma>0$ denotes the coefficient of surface tension, and $P_{0}$ is the constant atmospheric pressure. We suppose the bed is flat and given by $y=-H$ where $H$ is the mean depth of the wave. Now the nonslip boundary condition $v=0$ is applied to the bottom. Taking the curl of the momentum equations implies the conservation of vorticity along the particle path; that is, if we designate $\omega=v_{x}-v_{y}$ the vorticity equation is in the following form:

$$
\frac{\partial \omega}{\partial t}+u \frac{\partial \omega}{\partial x}+v \frac{\partial \omega}{\partial y}=0
$$

The mass equation (1) implies that there exists a stream function $\widetilde{\psi}$ such that $\widetilde{\varphi}_{x}=-v$ and $\widetilde{\varphi}_{y}=u$. Therefore, the vorticity can be expressed by the stream function by $\omega=-\Delta \widetilde{\varphi}$. In this paper, we assume $\omega$ is a constant initially. From the argument in (4), we know that if the initial voriticity is a constant then it remains constant all the time. Since we only consider solitary waves in this paper, we assume the uniform velocity on the surface as $x \rightarrow \pm \infty$. If moving the frame with this velocity, we can simply assume zero horizontal velocity in the far flow field on the free surface. Then we can reduce the problem to one for Laplace equation by introducing a new stream function $\varphi=\widetilde{\varphi}+(\omega / 2) y^{2}$, that is, $\Delta \varphi=0$. Consequently, one can introduce its harmonic conjugate $\phi$ satisfying

$$
\begin{aligned}
& \phi_{x}=\varphi_{y}=\tilde{\varphi}_{y}+\omega y=u+\omega y, \\
& \phi_{y}=-\varphi_{x}=-\widetilde{\varphi}_{x}=v .
\end{aligned}
$$

Now we can reformulate the boundary conditions by making use of $\phi$ and $\varphi$. The kinematic boundary conditions on the bottom and on the free surface take the following form:

$$
\begin{aligned}
\phi_{y} & =0 \quad \text { at } y=-H, \\
\zeta_{t} & =-\phi_{x} \zeta_{y}+\phi_{y}+\omega \zeta \zeta_{x} \text { at } y=\zeta(t, x)
\end{aligned}
$$

and the momentum equations now become

$$
\nabla\left[\phi_{t}+\frac{1}{2}|\nabla \tilde{\varphi}|^{2}+P+\omega \tilde{\varphi}+g y\right]=0 .
$$

Using (3), Bernoulli's Law, which is also referred to as the dynamical boundary condition, for the constant vorticity flow now becomes

$$
\phi_{t}+\frac{1}{2}\left(\phi_{x}^{2}+\phi_{y}^{2}\right)+\omega\left(\varphi-\zeta \phi_{x}\right)+g \zeta-\frac{\sigma}{\rho} \frac{\zeta_{x x}}{\left(1+\zeta_{x}^{2}\right)^{3 / 2}}=0
$$

Finally, we nondimensionalize the whole system by the time scale $\left(\sigma / \rho g^{3}\right)^{1 / 4}$, the length scale $(\sigma / \rho g)^{1 / 2}$, and the potential and steam scale $\left(\sigma^{3} / \rho^{3} g\right)^{1 / 4}$; therefore, with a little abuse of notations, the governing system for the constant vorticity flow takes the following form:

$$
\begin{aligned}
& \Delta \phi=0 \text { for }-H<y<\zeta(t, x), \\
& \phi_{y}=0 \text { for } y=-H, \\
& \zeta_{t}=-\phi_{x} \zeta_{x}+\phi_{y}+\omega \zeta \zeta_{x} \quad \text { for } y=\zeta(t, x), \\
& \phi_{t}+\frac{1}{2}\left(\phi_{x}^{2}+\phi_{y}^{2}\right)+\omega\left(\varphi-\zeta \phi_{x}\right) \\
& +\zeta-\frac{\zeta_{x x}}{\left(1+\zeta_{x}^{2}\right)^{3 / 2}}=0 \text { for } y=\zeta(t, x) .
\end{aligned}
$$

\section{Numerical Method}

Following the method presented in $[12,14,16]$ and the notations in [14], we introduce the time-dependent conformal mapping $z(\varepsilon, \eta, t)=x(\varepsilon, \eta, t)+i y(\varepsilon, \eta, t)$ which maps the physical domain $-H<y<\zeta(x, t)$ occupied by the fluid into a stripe $-H<\eta<0$. The following capital letters denote the values on the boundary $\eta=0$ :

$$
\begin{array}{ll}
X(\varepsilon, t)=x(\varepsilon, 0, t) & Y(\varepsilon, t)=y(\varepsilon, 0, t), \\
\Phi(\varepsilon, t)=\phi(\varepsilon, 0, t) & \Psi(\varepsilon, t)=\varphi(\varepsilon, 0, t) .
\end{array}
$$

Therefore the relations $X=\xi-\mathbb{C}[Y]$ and $\Phi=-\mathbb{C}[\Psi]$ hold, where $\mathbb{C}$ is a linear operator with the Fourier symbol $\widehat{\mathbb{C}}=$ $i \operatorname{coth}(H k)$. To derive the evolution equations for $\Phi$ and $Y$, 
the following equations from the chain rule of differentiation are useful:

$$
\begin{aligned}
\phi_{x} & =\frac{X_{\varepsilon} \Phi_{\varepsilon}+Y_{\varepsilon} \Psi_{\varepsilon}}{J}, \\
\phi_{y} & =\frac{Y_{\varepsilon} \Phi_{\varepsilon}-X_{\varepsilon} \Psi_{\varepsilon}}{J}, \\
Y_{\varepsilon} & =\zeta_{x} X_{\varepsilon} \Longrightarrow \zeta_{x}=\frac{Y_{\varepsilon}}{X_{\varepsilon}}, \\
Y_{t} & =\zeta_{x} X_{t}+\zeta_{t} \Longrightarrow \zeta_{t}=\frac{Y_{t} X_{\varepsilon}-X_{t} Y_{\varepsilon}}{X_{\varepsilon}}, \\
Y_{\varepsilon \varepsilon} & =\zeta_{x x}\left(X_{\varepsilon}\right)^{2}+\zeta_{\varepsilon} X_{\varepsilon \varepsilon} \Longrightarrow \zeta_{x x}=\frac{X_{\varepsilon} Y_{\varepsilon \varepsilon}-Y_{\varepsilon} X_{\varepsilon \varepsilon}}{X_{\varepsilon}^{3}},
\end{aligned}
$$

where $J=X_{\varepsilon}^{2}+Y_{\varepsilon}^{2}$. It follows from the kinematic boundary condition, the second equation of (6) and formulas (11), that

$$
X_{\varepsilon} Y_{t}-Y_{\varepsilon} X_{t}=-\Psi_{\varepsilon}+\omega Y Y_{\varepsilon}
$$

Notice that $z_{t} / z_{\varepsilon}$ is an analytic function of $\varepsilon+i \eta$ and it therefore follows that the real and imaginary parts of their boundary values are related by

$$
\begin{aligned}
& \operatorname{Im}\left(\frac{z_{t}}{z_{\varepsilon}}\right)_{\eta=0}=\frac{Y_{t} X_{\varepsilon}-X_{t} Y_{\varepsilon}}{J}=-\frac{\Psi_{\varepsilon}-\omega Y Y_{\varepsilon}}{J}, \\
& \operatorname{Re}\left(\frac{z_{t}}{z_{\varepsilon}}\right)_{\eta=0}=\frac{Y_{t} Y_{\varepsilon}+X_{t} X_{\varepsilon}}{J}=\mathbb{C}\left[\frac{\Psi_{\varepsilon}-\omega Y Y_{\varepsilon}}{J}\right] .
\end{aligned}
$$

In the curvilinear coordinates, the kinematic boundary condition becomes (13), while the dynamic boundary condition is obtained by substituting the expressions of (11) and (13) into Bernoulli's equation (8). Consider

$$
\begin{aligned}
Y_{t}= & Y_{\varepsilon} \mathbb{C}\left[\frac{\Psi_{\varepsilon}-\omega Y Y_{\varepsilon}}{J}\right]-X_{\varepsilon}\left(\frac{\Psi_{\varepsilon}-\omega Y Y_{\varepsilon}}{J}\right), \\
\Phi_{t}= & \frac{\Psi_{\varepsilon}^{2}-\Phi_{\varepsilon}^{2}}{2 J}-Y+\frac{X_{\varepsilon} Y_{\varepsilon \varepsilon}-Y_{\varepsilon} X_{\varepsilon \varepsilon}}{J^{3 / 2}} \\
& -\omega\left(\Psi-\frac{Y X_{\varepsilon} \Phi_{\varepsilon}}{J}\right)+\Phi_{\varepsilon} \mathbb{C}\left[\frac{\Psi_{\varepsilon}-\omega Y Y_{\varepsilon}}{J}\right] .
\end{aligned}
$$

Finally, (14) is a complete differential integral equation for updating $Y$ and $\Phi$.

\section{Results}

Due to the small length scale of the capillary-gravity waves $(\sim 1 \mathrm{~cm})$, deep water approximation is an appropriate assumption in this paper. Therefore the surface Euler system

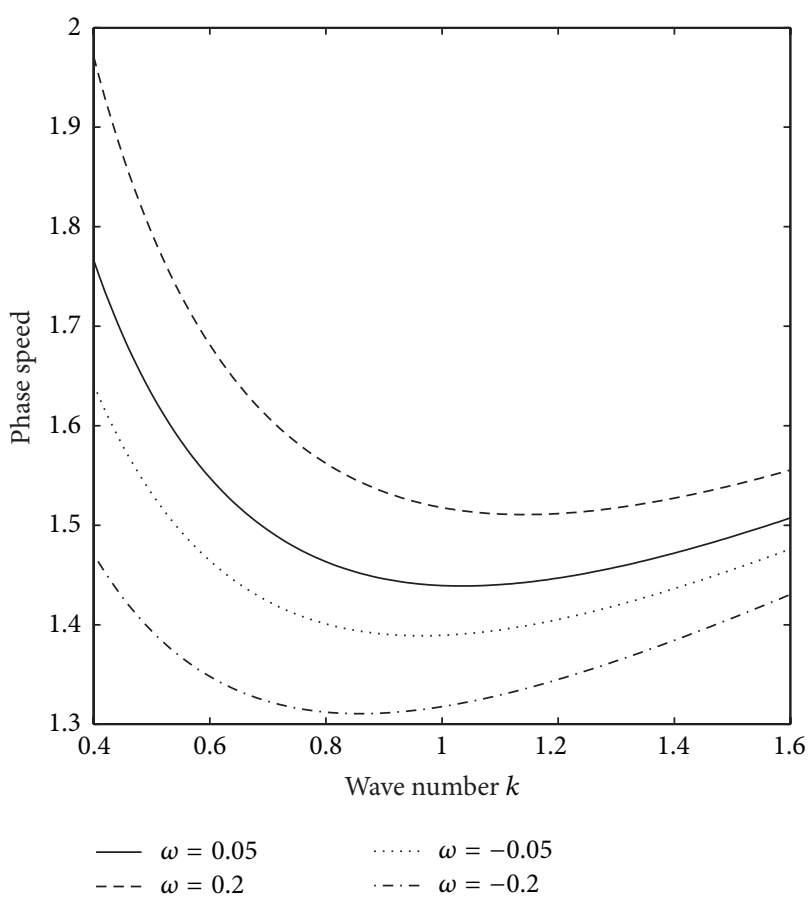

Figure 1: Dispersion relations for different vorticity strengths $\omega$. There is a local minimum of the phase speed for each curve.

in the curvilinear coordinates takes the following form:

$$
\begin{aligned}
X= & \varepsilon-H \mathbb{C}[Y], \\
\Psi= & H[\Phi], \\
Y_{t}= & Y_{\varepsilon} H\left[\frac{\Psi_{\varepsilon}-\omega Y Y_{\varepsilon}}{J}\right]-X_{\varepsilon}\left(\frac{\Psi_{\varepsilon}-\omega Y Y_{\varepsilon}}{J}\right), \\
\Phi_{t}= & \frac{\Psi_{\varepsilon}^{2}-\Phi_{\varepsilon}^{2}}{2 J}-Y+\frac{X_{\varepsilon} Y_{\varepsilon \varepsilon}-Y_{\varepsilon} X_{\varepsilon \varepsilon}}{J^{3 / 2}}-\omega\left(\Psi-\frac{Y X_{\varepsilon} \Phi_{\varepsilon}}{J}\right) \\
& +\Phi_{\varepsilon} H\left[\frac{\Psi_{\varepsilon}-\omega Y Y_{\varepsilon}}{J}\right],
\end{aligned}
$$

where $H$ is the famous Hilbert transform with the Fourier symbol $i \operatorname{sgn}(k)$. There are three well-known conservative quantities for the evolution system: conservation of mass, momentum, and energy. Consider

$$
\text { mass }=\int Y X_{\varepsilon} d \varepsilon \text {, }
$$

momentum $=\int \Phi_{\varepsilon} Y d \varepsilon-\int \frac{\omega}{2} Y^{2} X_{\varepsilon} d \varepsilon$,

energy $=\frac{1}{2} \int\left[\Phi_{\varepsilon} \Psi+\frac{\omega^{2} Y^{3} X_{\varepsilon}}{3}-\omega Y^{2} \Phi_{\varepsilon}+2\left(\sqrt{J}-X_{\varepsilon}\right)\right] d \varepsilon$. 


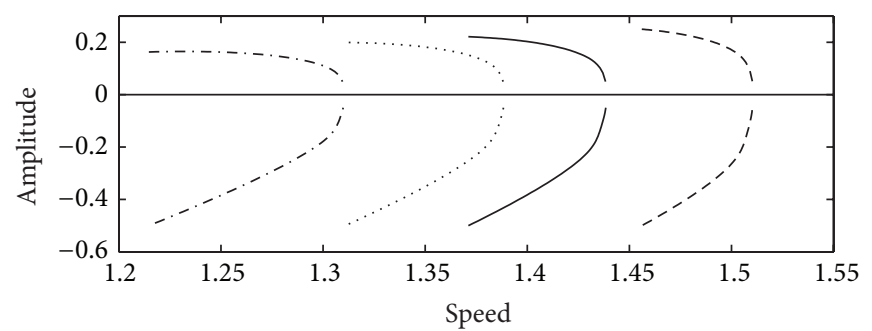

(a)

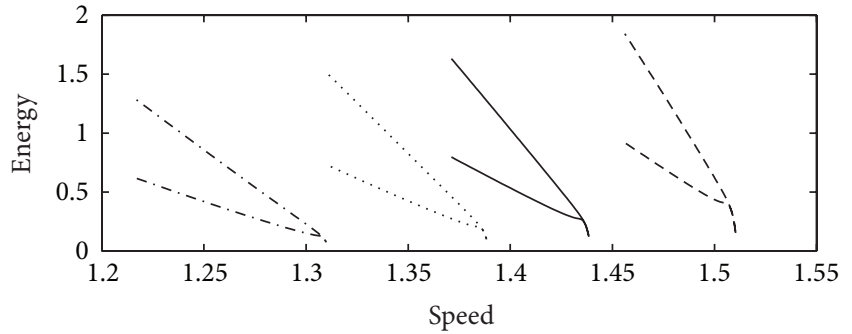

(b)

Figure 2: (a) Amplitude versus speed bifurcation. There are two branches for each $\omega$ and bifurcate from the minimum of the phase speed. From left to right: $\omega=-0.2,-0.05,0.05,0.2$. (b) Energy versus speed curves for different $\omega$. The upper branches are for elevation solitary wave, and the lower branches are for depression ones. From left to right: $\omega=-0.2,-0.05,0.05,0.2$, respectively.

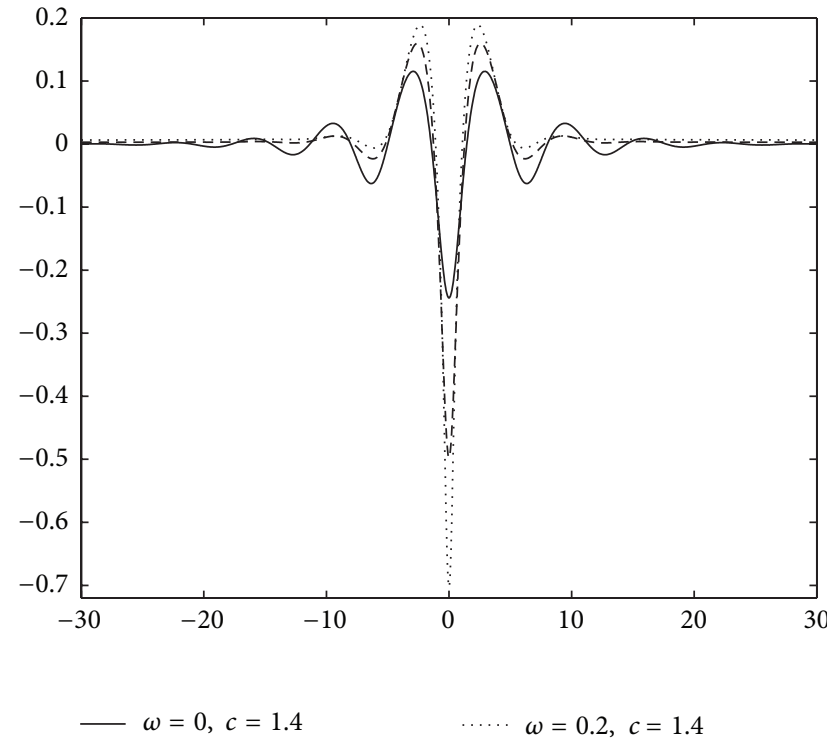

(a)

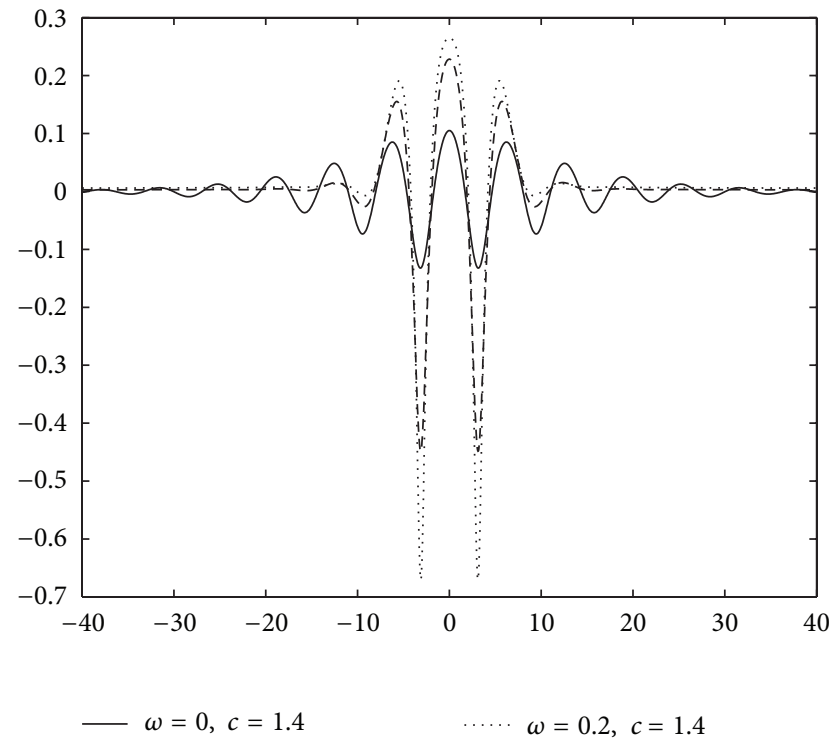

(b)

FIGURE 3: Wave profiles with the same translating speed but different vorticity. Depression solitary waves at $c=1.4$ (a): $\omega=0$ (solid line), $\omega=0.1$ (dashed line), and $\omega=0.2$ (dotted line); elevation solitary waves at $c=1.41(\mathrm{~b}): \omega=0$ (solid line), $\omega=0.1$ (dashed line), and $\omega=0.2$ (dotted line).

If we linearize the system (15), it is easy to obtain the dispersion relation between the wave number $k$ and the frequency $\Omega$ which results in the following phase speed:

$$
\begin{aligned}
& \Omega^{2}-\omega \operatorname{sgn}(k) \Omega-|k|\left(1+k^{2}\right)=0 \\
& \Longrightarrow \text { phase speed } \triangleq \frac{\Omega}{k}=\frac{\omega+\sqrt{\omega^{2}+4|k|\left(1+k^{2}\right)}}{2 k} .
\end{aligned}
$$

The linear dispersion relation is presented in Figure 1, for different vorticity strengths. The plotting of the phase speed versus wave number shows that there is a local minimum for each curve, and it has been figured out in [16] that solitary waves exist only below this point, and two branches of solitary waves bifurcate from the minimum of the phase speed (see Figure 3).
4.1. Traveling Wave. To study the dynamics of the capillarygravity solitary waves with linear background shear, it is the first step to seek traveling wave solution to the Euler equations (9) with wave propagating speed $c$; we assume all functions depend on $x-c t$; then, the boundary conditions on the free surface read

$$
\begin{gathered}
-c \zeta_{x}=-\phi_{x} \zeta_{x}+\phi_{y}+\omega \zeta \zeta_{x} \\
-c \phi_{x}+\frac{1}{2}\left[\phi_{x}^{2}+\phi_{y}^{2}\right]+\omega\left(\psi-\zeta \phi_{x}\right)+\zeta-\frac{\zeta_{x x}}{\left(1+\zeta_{x}^{2}\right)^{3 / 2}}=0 .
\end{gathered}
$$

In the curvilinear coordinates, (18) can be rewritten as follows:

$$
\Psi_{\varepsilon}=c Y_{\varepsilon}+\omega Y Y_{\varepsilon} \Longrightarrow \Psi=c Y+\frac{\omega}{2} Y^{2}
$$




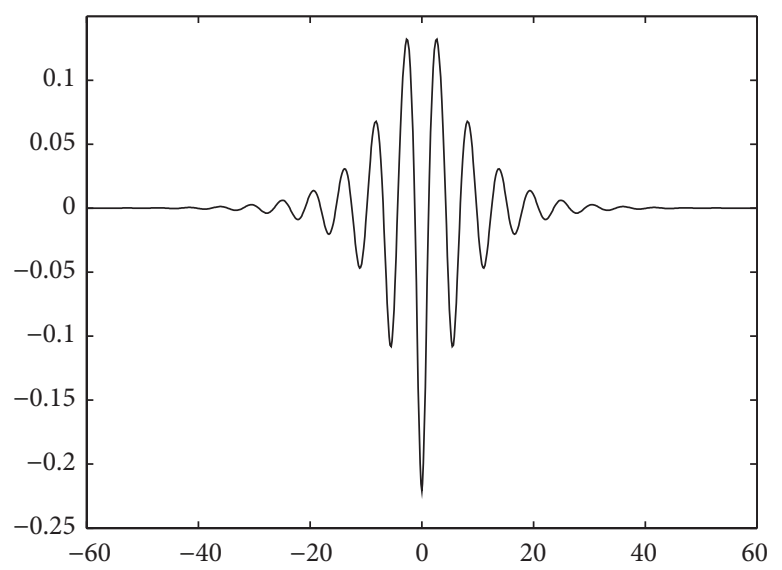

(a)

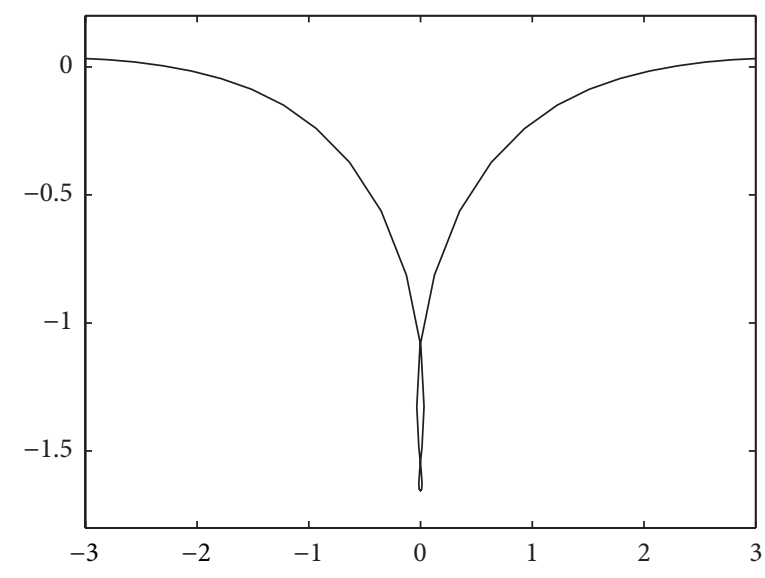

(c)

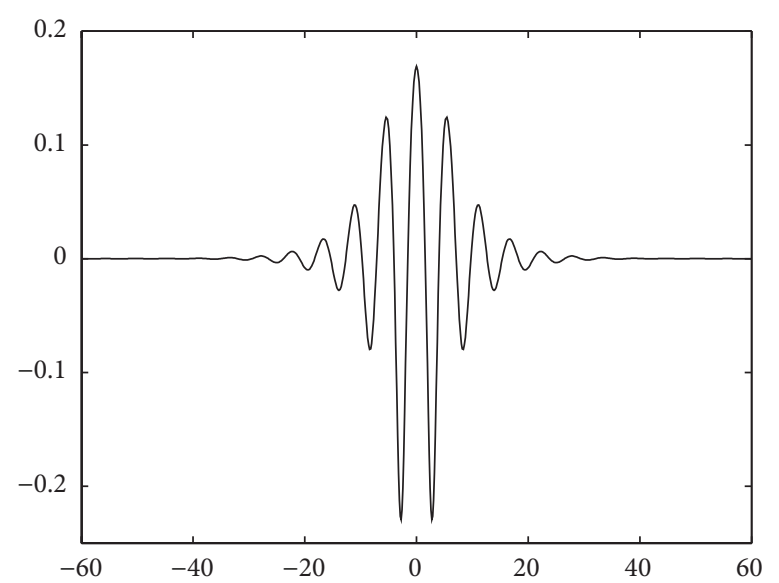

(b)

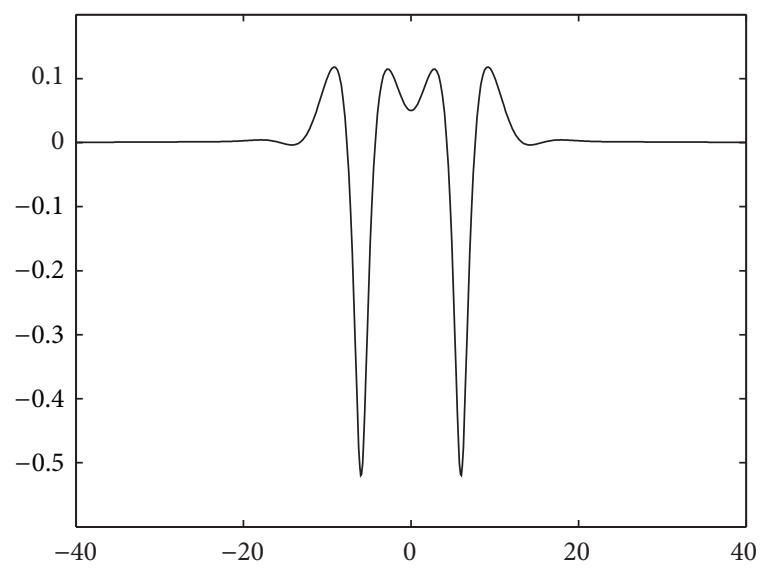

(d)

FIGURE 4: Typical profiles of two branches capillary-gravity solitary waves. Depression solitary wave (a) with $y(0)=-0.2210, c=1.5041$, and $\omega=0.2$; elevation solitary wave (b) with $y(0)=0.1692, c=1.5005$, and $\omega=0.2$. Extreme configuration of the depression solitary wave (c) with $y(0)=-1.7793, c=0.5$, and $\omega=-0.2$; large amplitude elevation solitary wave (d) with $y(0)=0.05, c=1.2093$, and $\omega=-0.2$.

Similarly, the dynamic boundary condition (19) can be simplified as follows:

$$
\frac{\left[\omega H\left(Y Y_{\varepsilon}\right)+c+\omega Y X_{\varepsilon}\right]^{2}}{2 J}-\frac{c^{2}}{2}+Y-\frac{X_{\varepsilon} Y_{\varepsilon \varepsilon}-Y_{\varepsilon} X_{\varepsilon \varepsilon}}{J^{3 / 2}}=0
$$

Equation (21) only depends on the variable $Y$ which is convenient for numerical simulation. As a special case, for irrotational capillary-gravity wave, (21) recovers (12) in [14]. If ignoring the surface tension, this equation for traveling wave is equivalent to the equation solved by Choi [13] under a different scaling.

The nonlinear integral-differential equation (21) is discretized in the Fourier space using 1024 modes and solved by Newton's method and continuation method. In Figure 2, bifurcation pictures are shown for different $\omega$. For each $\omega$, solitary waves are found below the minimum of the phase speed, and small $\omega$ has small minimum phase speed. There are two branches of solitary waves-elevation wave with the center above zero and depression wave with the center below zero bifurcating from the minimum of the phase speed. For large depression solitary waves, limit profile of selfintersect configuration is numerically found for nonzero $\omega$. This unphysical profile has already been found in [16] for the irrotational wave. Large amplitude depression solitary wave which is composed of two depression solitary waves is also found for the flow with shear background (see Figure 2).

4.2. Stability. For irrotational capillary-gravity solitary waves, the stability analysis has been carried out by Calvo and Akylas (2002), and later by Milewski et al. [14]. In this section, stability properties are examined by time-dependent numerical experiments for the capillary-gravity solitary waves when the linear background shear is added, and similar results to irrotational case are observed. Small and moderate depression solitary waves are proved to be stable in our numerical experiments. However elevation solitary waves show different stability behaviors (see Figure 4). Small amplitude elevation wave is unstable due to small perturbation, and it finally evolves into a stable depression solitary wave with some radiations. Large amplitude elevation 

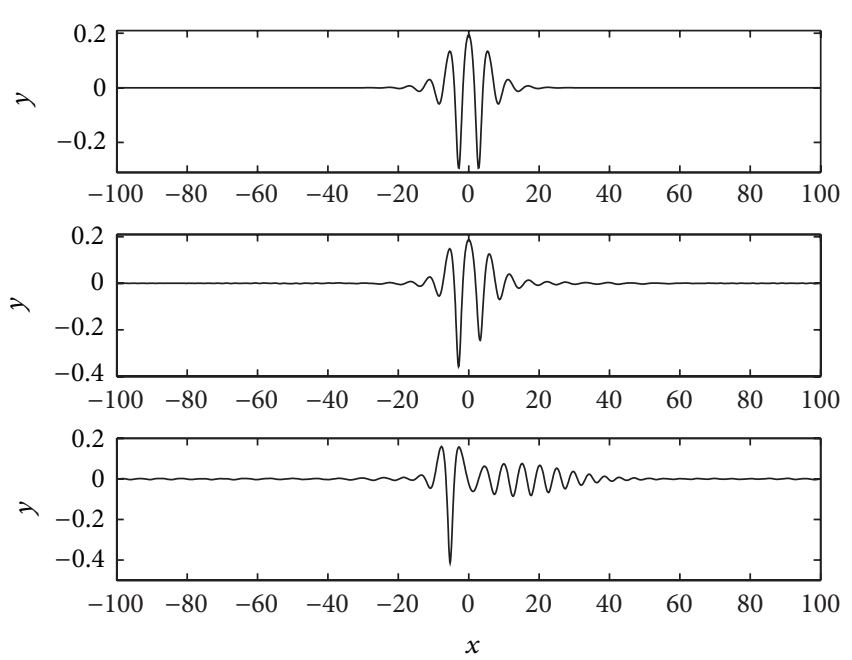

(a)
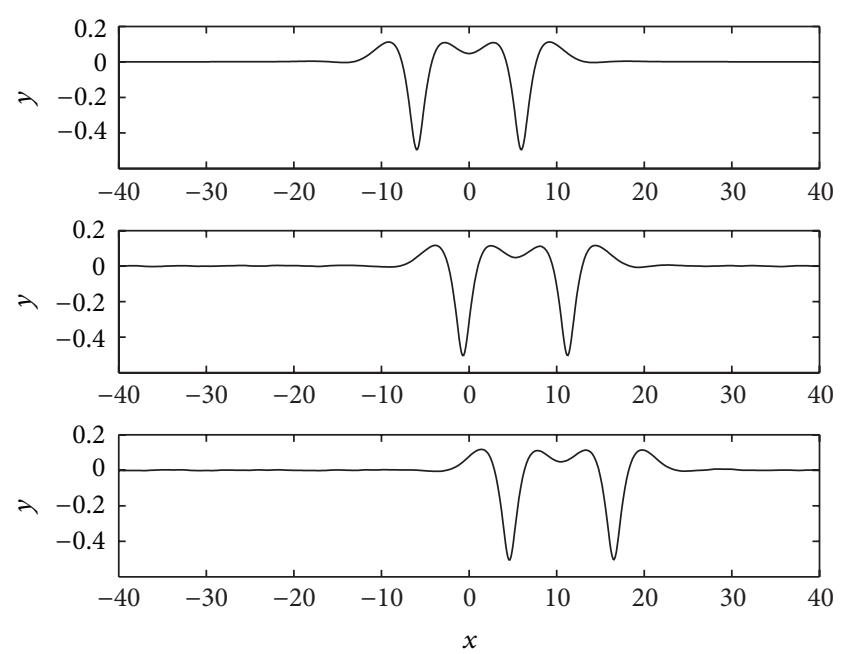

(b)

Figure 5: (a) Unstable elevation solitary wave with $y(0)=0.2047, c=1.4897$, and $\omega=0.2 .5 \%$ amplitude-decreasing perturbation is applied, and it finally evolves into a depression wave with some radiations. The plots are shown for $t=0,125,300$ from top to bottom; (b) stable large amplitude elevation wave with $y(0)=0.05, c=1.2093$, and $\omega=-0.2 .5 \%$ amplitude-decreasing perturbation is applied, $t=0,1000,2000$ from top to bottom. The frame is moved with the original propagating speed $c$.

solitary wave is stable, and no significant change happens even for long time numerical simulation (see Figure 5).

\section{Conclusions}

Numerical experiments for the dynamics of the capillarygravity solitary waves on a linear shear current are performed in the first time. The bifurcation pictures and two branches of solitary waves which are qualitatively similar to the irrotational capillary-gravity solitary waves are found. The bifurcation points are found to be monotonic with the shear strengths. Stability properties of these branches are tested using the numerical evolution equation based on the timedependent conformal map technique. Finally, both head on collisions and overtaking collisions are computed mainly for the case of one elevation wave and one depression wave, which are firstly performed to the best of our knowledge.

\section{Conflict of Interests}

The authors declare that there is no conflict of interests regarding the publication of this paper.

\section{Acknowledgments}

The authors would like to thank the reviewers for providing them with constructive comments and suggestions. This work is supported by Open Fund (PLN1004) of State Key Laboratory of Oil and Gas Reservoir Geology and Exploitation (Southwest Petroleum University).

\section{References}

[1] J. A. Simmen, Steady deep-water waves on a linear shear current [Ph.D. thesis], 1984.

[2] A. F. Teles da Silva and D. H. Peregrine, "Steep, steady surface waves on water of finite depth with constant vorticity," Journal of Fluid Mechanics, vol. 195, pp. 281-302, 1988.

[3] J.-M. Vanden-Broeck, "Steep solitary waves in water of finite depth with constant vorticity," Journal of Fluid Mechanics, vol. 274, pp. 339-348, 1994.

[4] J.-M. Vanden-Broeck, "Periodic waves with constant vorticity in water of infinite depth," IMA Journal of Applied Mathematics, vol. 56, no. 3, pp. 207-217, 1996.

[5] A. Constantin, D. Sattinger, and W. Strauss, "Variational formulations for steady water waves with vorticity," Journal of Fluid Mechanics, vol. 548, pp. 151-163, 2006.

[6] A. Constantin and W. Strauss, "Exact steady periodic water waves with vorticity" Communications on Pure and Applied Mathematics, vol. 57, no. 4, pp. 481-527, 2004.

[7] J. Ko and W. Strauss, "Effect of vorticity on steady water waves," Journal of Fluid Mechanics, vol. 608, pp. 197-215, 2008.

[8] E. Wahlén, "Steady periodic capillary-gravity waves with vorticity," SIAM Journal on Mathematical Analysis, vol. 38, no. 3, pp. 921-943, 2006.

[9] J. Biello and J. K. Hunter, "Nonlinear Hamiltonian waves with constant frequency and surface waves on vorticity discontinuities," Communications on Pure and Applied Mathematics, vol. 63, no. 3, pp. 303-336, 2010.

[10] A. Constantin, R. I. Ivanov, and E. M. Prodanov, "NearlyHamiltonian structure for water waves with constant vorticity," Journal of Mathematical Fluid Mechanics, vol. 10, no. 2, pp. 224237, 2008.

[11] E. Wahlén, "A Hamiltonian formulation of water waves with constant vorticity," Letters in Mathematical Physics, vol. 79, no. 3, pp. 303-315, 2007. 
[12] A. I. Dyachenko, V. E. Zakharov, and E. A. Kuznetsov, "Nonlinear dynamics of the free surface of an ideal fluid," Plasma Physics Reports, vol. 22, no. 10, pp. 829-840, 1996.

[13] W. Choi, "Nonlinear surface waves interacting with a linear shear current," Mathematics and Computers in Simulation, vol. 80, no. 1, pp. 29-36, 2009.

[14] P. A. Milewski, J.-M. Vanden-Broeck, and Z. Wang, "Dynamics of steep two-dimensional gravity-capillary solitary waves," Journal of Fluid Mechanics, vol. 664, pp. 466-477, 2010.

[15] Y. Kang and J.-M. Vanden-Broeck, "Gravity-capillary waves in the presence of constant vorticity," European Journal of Mechanics, B, vol. 19, no. 2, pp. 253-268, 2000.

[16] J.-M. Vanden-Broeck and F. Dias, "Gravity-capillary solitary waves in water of infinite depth and related free-surface flows," Journal of Fluid Mechanics, vol. 240, pp. 549-557, 1992. 


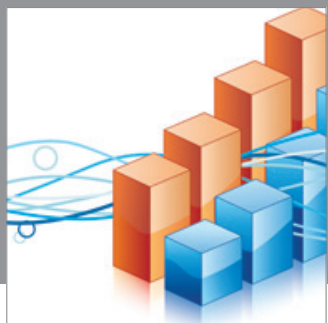

Advances in

Operations Research

mansans

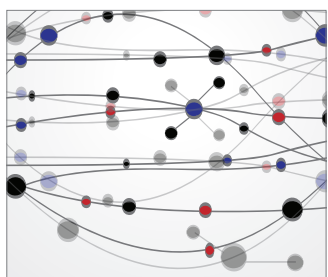

The Scientific World Journal
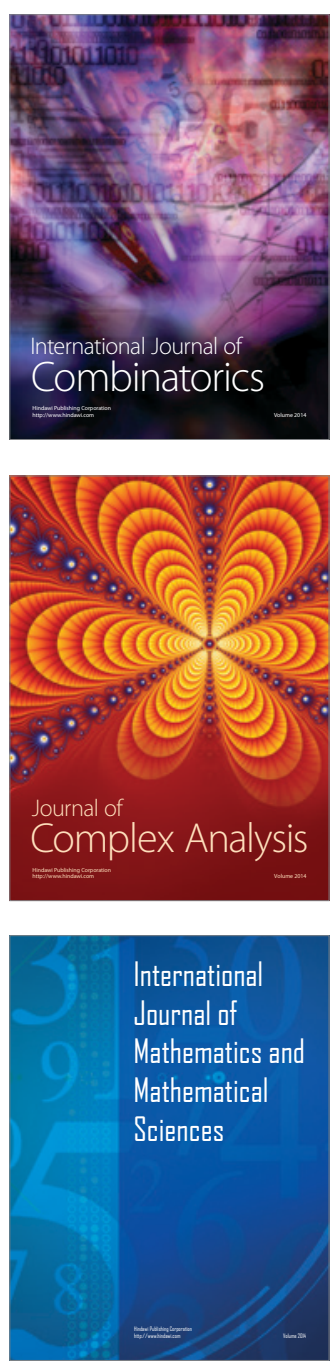
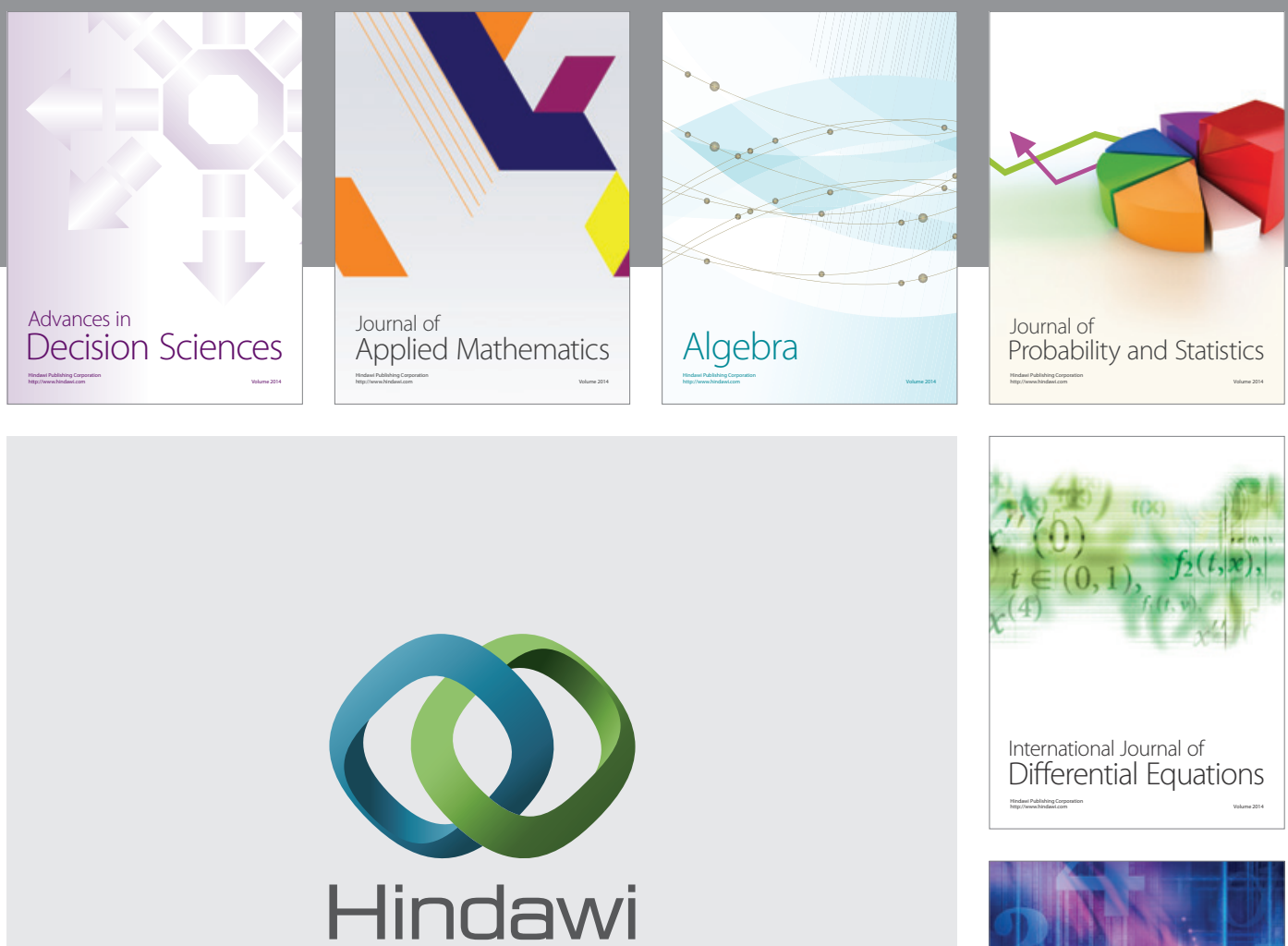

Submit your manuscripts at http://www.hindawi.com
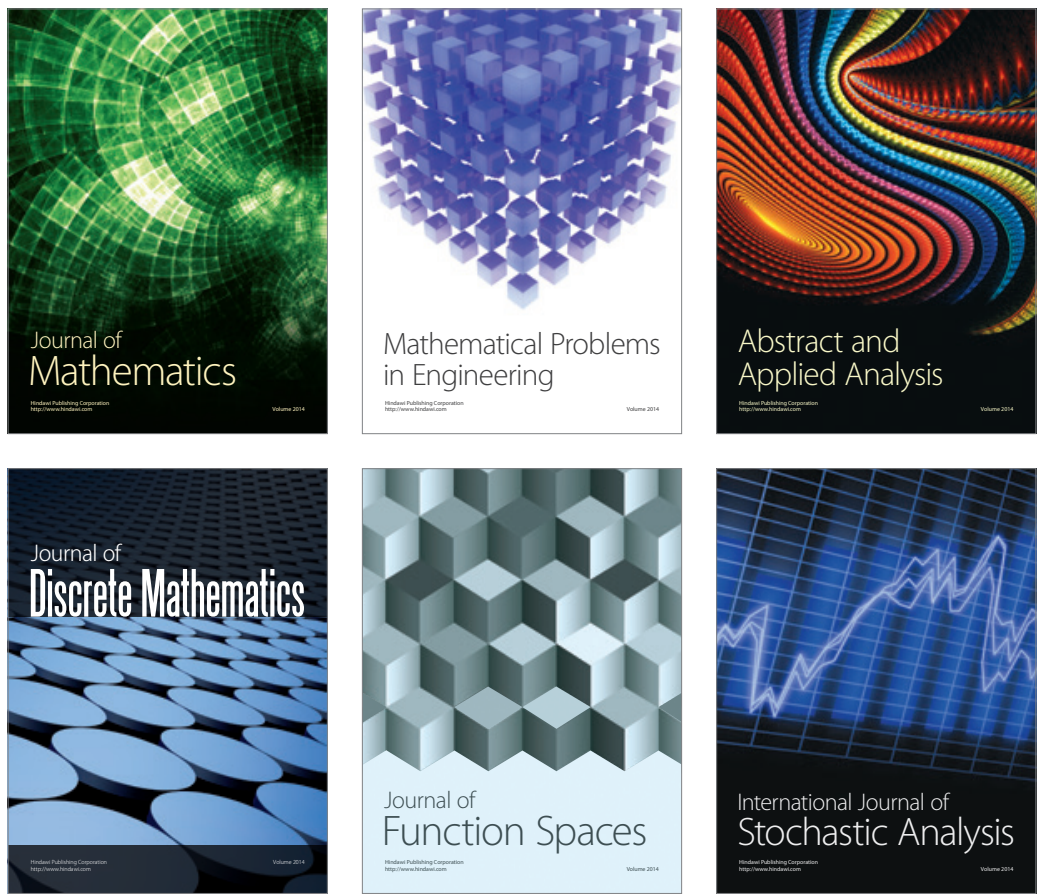

Journal of

Function Spaces

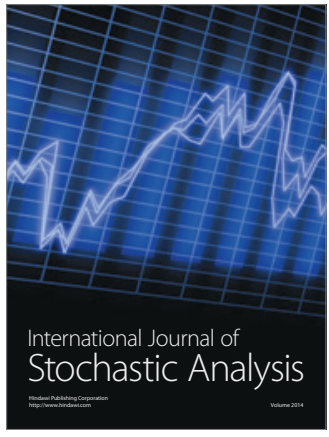

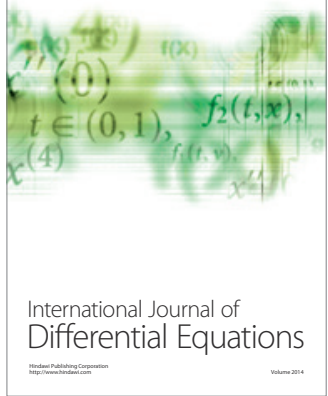
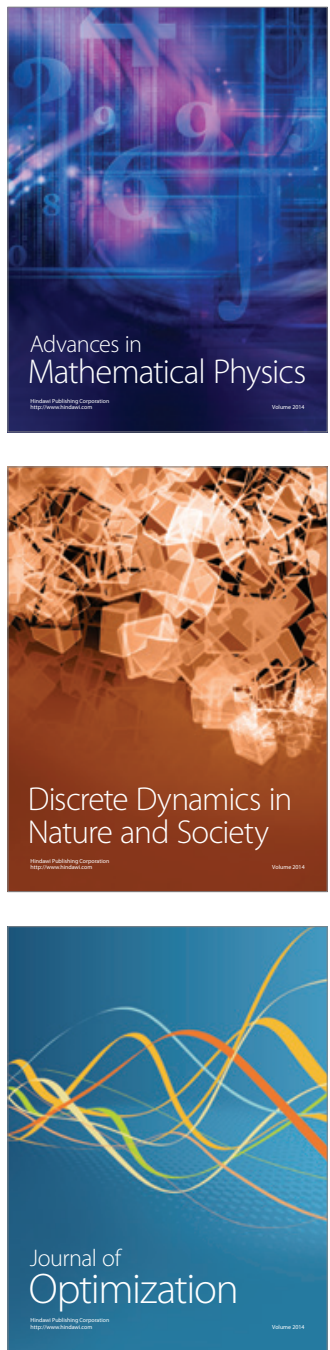